\title{
Estimations of Solutions of the Sturm- Liouville Equation with Respect to a Spectral Parameter
}

\author{
Łukasz Rzepnicki
}

\begin{abstract}
This paper is concerned with estimations of solutions of the Sturm-Liouville equation

$$
\left(p(x) y^{\prime}(x)\right)^{\prime}+\left(\mu^{2}-2 i \mu d(x)-q(x)\right) \rho(x) y(x)=0, \quad x \in[0,1],
$$

where $\mu \in \mathbb{C}$ is a spectral parameter. We assume that the strictly positive function $\rho \in L_{\infty}[0,1]$ is of bounded variation, $p \in W_{1}^{1}[0,1]$ is also strictly positive, while $d \in L_{1}[0,1]$ and $q \in L_{1}[0,1]$ are real functions. The main result states that for any $r>0$ there exists a constant $c_{r}$ such that for any solution $y$ of the Sturm-Liouville equation with $\mu$ satisfying $|\operatorname{Im} \mu| \leq r$, the inequality $\|y(\cdot, \mu)\|_{C} \leq c_{r}\|y(\cdot, \mu)\|_{L_{1}}$ is true. We apply our results to a problem of vibrations of an inhomogeneous string of length one with damping, modulus of elasticity and potential, rewritten in an operator form. As a consequence, we obtain that the operator acting on a certain energy Hilbert space is the generator of an exponentially stable $C_{0}$-semigroup.
\end{abstract}

Mathematics Subject Classification (2010). Primary 34B24; Secondary 47B44, 47D03.

Keywords. Sturm-Liouville equation, damped string, one-dimensional wave equation, exponentially stable $C_{0}$-semigroup, Hilbert space.

\section{Introduction}

The investigation of numerous physical problems requires the analysis of various spectral boundary-value problems of Sturm-Liouville type, which explains the necessity of studying estimations or asymptotic with respect to a spectral parameter of their solutions. For an extensive bibliography on these topics, see [6]. In particular, in recent years much attention has been given to the investigation of non-self-adjoint spectral problems related to oscillations of an inhomogeneous string under various conditions of damping, potential, 
etc. (see, e.g., [10] and the references therein, or $[1,11,15,18,22]$ ). This problem can be used as a model for much more complicated phenomena in physics such as vibrating strings and membranes in vehicle dynamics or aircraft.

The focus of the present work is on the equation

$$
l_{\mu}(y):=\left(p(x) y^{\prime}(x)\right)^{\prime}+\left(\mu^{2}-2 i \mu d(x)-q(x)\right) \rho(x) y(x)=0, \quad x \in[0,1] .
$$

In what follows we write $C=C[0,1]$ as the space of continuous functions with the supremum norm $\|\cdot\|_{C}$. The symbol $W_{p}^{k}[0,1]$, where $p \geq 1$ and $k \in \mathbb{N}$, stands for the Sobolev space with the $k$-th derivative in $L_{p}[0,1]$ which is a classical Lebesgue space. For convenience, we introduce the notation

$\widehat{W}_{2}^{1}[0,1]:=\left\{y \in W_{2}^{1}[0,1] ; y(0)=0\right\}, \widehat{W}_{1}^{2}[0,1]:=\left\{y \in W_{1}^{2}[0,1] ; y(0)=0\right\}$.

We assume that the real function $\rho$ satisfies

$$
\rho \in L_{\infty}[0,1], \quad 0<m_{\rho} \leq \rho(x) \leq M_{\rho} \text { for a.e. } x \in[0,1]
$$

and is of bounded variation. The remaining functions are real valued and satisfy

$$
\begin{aligned}
& p \in W_{1}^{1}[0,1], \quad 0<m_{p} \leq p(x) \leq M_{p}, \quad x \in[0,1] . \\
& d \in L_{1}[0,1] \\
& q \in L_{1}[0,1] .
\end{aligned}
$$

Note that in general we can not require that the solution $y$ of (1.1) is from $W_{2}^{2}[0,1]$ since $d$ and $q$ belong to $L_{1}[0,1]$. The assumption (1.3) allows us to define the solution of (1.1) as follows (see [24, Definition 2.2.1]):

Definition 1.1. A solution of the Eq. (1.1) is a complex valued function $y \in W_{1}^{2}[0,1]$ which satisfies (1.1) almost everywhere.

The main result of this paper, proven in Sect. 2, states that:

Theorem 1.2. Let $p, d$ and $q$ satisfy the assumptions (1.3), (1.4) and (1.5), respectively. Moreover, let $\rho$ be as in (1.2) and of bounded variation. Then for any $r>0$ there exists a constant $c_{r}$ such that for all $\mu$ with $|\operatorname{Im} \mu| \leq r$ and for any solution $y$ of (1.1), the following estimation holds

$$
\|y(\cdot, \mu)\|_{C[0,1]} \leq c_{r}\|y(\cdot, \mu)\|_{L_{1}[0,1]} \cdot
$$

What is more, we obtain the corollary concerning estimations for solutions of Eq. (1.1) with boundary conditions

$$
y(0)=0, \quad y^{\prime}(0)=1,
$$

which states that

$$
\|y(\cdot, \mu)\|_{C} \leq \frac{c}{|\mu|} \exp \left(m_{p}^{-1 / 2}|\tau|\left\|\rho^{1 / 2}\right\|_{L_{1}}\right),
$$

where $\mu=s+i \tau$ and $|\mu|>1$.

If we consider the Eq. (1.1) with $d=0$ and appropriate self-adjoint boundary conditions, then the estimation (1.6) implies uniform boundedness 
of normed eigenfunctions $y_{k}, k=1,2, \ldots$ of the aforementioned spectral problem with the spectral parameter $\lambda=\mu^{2}$, i.e.,

$$
\sup _{k}\left\|y_{k}\right\|_{C} \leq c, \quad\left\|y_{k}\right\|_{L_{2}}=1
$$

The question of uniform boundedness as in (1.8), with many different boundary conditions and assumptions on the coefficient functions was extensively investigated, see e.g. [7-9].

The Eq. (1.1) occurs naturally in the study of vibrations of an inhomogeneous string of length one with density $\rho$, viscous damping $d$, modulus of elasticity $p$ and potential $q$. We will consider a string with one end fixed and damped with complex parameter $h$ into another. This problem with appropriate assumptions can be transformed into an abstract Cauchy problem for a closed, densely defined operator $B_{h}$ acting on an energy Hilbert space $H$. Then eigenfunctions of $B_{h}$ are connected with solutions of the equation $l_{\mu}(y)=0$ with appropriate boundary conditions. In Sect. 3 we will apply the estimations obtained in Sect. 2 to prove that $B_{h}$ is the generator of an exponentially stable $C_{0}$-semigroup of contractions. Additionally, we provide the explicit, positive lower bound of the real part of its spectrum.

The results of Sect. 2 generalize those of [12, Theorem 3.1], where the Eq. (1.1) was considered with $p=1, d=0$ and $q=0$. The content of Sect. 3 extends theorems obtained in [19] also in a simpler case.

\section{Estimations with Respect to a Spectral Parameter}

The proof of Theorem 1.2 depends on some auxiliary estimations, the most important of which are stated in the lemma below. This lemma, apart from being the main tool in the proof of Theorem 1.2, is also useful in many applications as we will show in the next section.

Lemma 2.1. Let $p, d$ and $q$ satisfy the assumptions (1.3), (1.4) and (1.5), respectively. Moreover, let $\rho$ be as in (1.2) and of bounded variation $\mathcal{V}=\mathcal{V}(\rho)$. Given $|\mu|>1$, any solution $y$ of (1.1) and $x \in[0,1]$, the following estimations are true:

$$
\begin{aligned}
& \left(|\mu|^{2}|y(0)|^{2}+\left|y^{\prime}(0)\right|^{2} m_{p} / M_{\rho}\right) \exp \left(-2 \alpha_{0}(|\tau|)\right) \\
& \quad \leq|\mu|^{2}|y(x)|^{2}+\left|y^{\prime}(x)\right|^{2} M_{p} / m_{\rho}, \\
& \left(|\mu|^{2}|y(x)|^{2}+\left|y^{\prime}(x)\right|^{2} m_{p} / M_{\rho}\right) \\
& \quad \leq\left(|\mu|^{2}|y(0)|^{2}+\left|y^{\prime}(0)\right|^{2} M_{p} / m_{\rho}\right) \exp \left(2 \alpha_{0}(|\tau|)\right),
\end{aligned}
$$

where $\tau=\operatorname{Im} \mu$ and $m_{g}=m_{p} m_{\rho}$, and

$$
\alpha_{0}(|\tau|)=|\tau| \frac{\left\|\rho^{1 / 2}\right\|_{L_{1}}}{m_{p}^{1 / 2}}+\frac{M_{p} \mathcal{V}+M_{\rho}\left\|p^{\prime}\right\|_{L_{1}}}{2 m_{g}}+\frac{M_{\rho}}{m_{g}^{1 / 2}}\left(\|q\|_{L_{1}}+\|d\|_{L_{1}}\right) .
$$

The proof of Lemma 2.1 is taken care of two steps. The first is the transformation as in [13] of the Eq. (1.1) into a system of first order differential 
equations (see also [12]). Then we will apply the following proposition, which is a particular case of [3, Section $3, \S 4]$.

Proposition 2.2. Let $M=M(x)$, where $x \in[0,1]$, be a $2 \times 2$ matrix-valued function with entries $a_{i j} \in L_{1}[0,1]$. Moreover, let $\alpha_{ \pm}=\alpha_{ \pm}(x)$ be the eigenvalues of the self-adjoint matrix $M_{R}(x)=\left(M(x)+M^{*}(x)\right) / 2$ with $\alpha_{-}(x) \leq \alpha_{+}(x)$ a.e.. Then for any solution $Y$ of the vector differential equation

$$
Y^{\prime}(x)=M(x) Y(x), \quad Y(x)=\left[y_{1}(x), y_{2}(x)\right]^{T}, \quad x \in[0,1],
$$

the following estimations hold for any $x \in[0,1]$

$$
\|Y(0)\| \exp \left(\int_{0}^{x} \alpha_{-}(t) d t\right) \leq\|Y(x)\| \leq\|Y(0)\| \exp \left(\int_{0}^{x} \alpha_{+}(t) d t\right),
$$

where $\|\cdot\|$ denotes the Euclidean norm in $\mathbb{C}^{2}:\left\|\left[y_{1}, y_{2}\right]^{T}\right\|^{2}=\left|y_{1}\right|^{2}+\left|y_{2}\right|^{2}$.

The second step involves estimations of the eigenvalues $\alpha_{ \pm}$. This part requires some knowledge about approximation of functions from $L_{1}[0,1]$ by functions from $W_{1}^{1}[0,1]$.

Proposition 2.3. [12, Proposition 1.1] For every $f \in L_{1}[0,1]$ there exists a family of functions $f_{\delta} \in W_{1}^{1}[0,1]$ where $\delta \in(0,1)$, such that the following estimates are true

$$
\left\|f-f_{\delta}\right\|_{L_{1}} \leq 3 \omega_{1}(f, \delta), \quad\left\|f_{\delta}^{\prime}\right\|_{L_{1}} \leq \delta^{-1} \omega_{1}(f, \delta),
$$

where

$$
\omega_{1}(g, \epsilon)=\sup _{0<\xi \leq \epsilon}\|g(\cdot+\xi)-g(\cdot)\|_{L_{1}[0,1-\xi]}, \quad \epsilon \in(0,1), \quad g \in L_{1}[0,1],
$$

is the integral modulus of continuity, with $\omega_{1}(g, \epsilon) \rightarrow 0$ when $\epsilon \rightarrow 0$.

Moreover, if $f \in L_{\infty}[0,1]$ satisfies $m \leq f(x) \leq M$ a.e., then for an arbitrary $\delta \in(0,1)$ and $x \in[0,1]$ we have the inequality $m \leq f_{\delta}(x) \leq M$.

We now move on to the proof of Lemma 2.1.

Proof. First consider the case when $\rho \in L_{1}[0,1]$ has the representation

$$
\rho(x)=\eta(x)+\beta(x), \quad \eta \in W_{1}^{1}[0,1], \quad \beta \in L_{1}[0,1],
$$

where

$$
0<m_{\eta} \leq \eta(x) \leq M_{\eta}, \quad x \in[0,1] .
$$

Therefore, only $\eta$ is bounded, not $\rho$ itself. Let $y=y(x, \mu)$ be a solution of (1.1) for $\mu \neq 0$. Denote

$$
g=p \eta \in W_{1}^{1}[0,1], \quad b=\sqrt{\frac{\eta}{p}}=\frac{\sqrt{g}}{p} \in W_{1}^{1}[0,1],
$$

so that

$$
m_{g} \leq g(x) \leq M_{g}, \quad m_{b} \leq b(x) \leq M_{b}, \quad x \in[0,1]
$$

where the positive constants are

$$
m_{g}=m_{p} m_{\eta}, \quad M_{g}=M_{p} M_{\eta}, \quad m_{b}=\left(m_{\eta} / M_{p}\right)^{1 / 2}, \quad M_{b}=\left(M_{\eta} / m_{p}\right)^{1 / 2} .
$$


Let $y$ be a solution of (1.1). Define the functions $w_{j}=w_{j}(x, \mu)$ for $j=1,2$ by setting

$$
w_{1}=\frac{1}{2}\left(y+\frac{y^{\prime}}{i \mu b}\right), \quad w_{2}=\frac{1}{2}\left(y-\frac{y^{\prime}}{i \mu b}\right) .
$$

Then the following relations are true:

$$
y=w_{1}+w_{2}, \quad p y^{\prime}=i \mu \sqrt{g}\left(w_{1}-w_{2}\right) .
$$

Differentiating (2.10), using both the fact that $y$ is a solution of (1.1) and the identities (2.11), we obtain a system of first order differential equations for $Y(x, \mu)=\left[w_{1}(x, \mu), w_{2}(x, \mu)\right]^{T}$ :

$$
Y^{\prime}(x, \mu)=M(x, \mu) Y(x, \mu), \quad M(x, \mu)=\left[a_{i j}(x, \mu)\right]_{i, j=1}^{2},
$$

where the matrix $M$ consists of the elements from $L_{1}[0,1]$ given by:

$$
\begin{aligned}
& a_{11}(x, \mu)=i \mu b(x)-\frac{g^{\prime}(x)}{4 g(x)}+\frac{d(x) \rho(x)}{\sqrt{g(x)}}-i \frac{q(x) \rho(x)}{2 \mu \sqrt{g(x)}}+\frac{i \mu \beta(x)}{2 \sqrt{g(x)}} \\
& a_{12}(x, \mu)=\frac{g^{\prime}(x)}{4 g(x)}+\frac{d(x) \rho(x)}{\sqrt{g(x)}}-i \frac{q(x) \rho(x)}{2 \mu \sqrt{g(x)}}+\frac{i \mu \beta(x)}{2 \sqrt{g(x)}} \\
& a_{21}(x, \mu)=\frac{g^{\prime}(x)}{4 g(x)}-\frac{d(x) \rho(x)}{\sqrt{g(x)}}+i \frac{q(x) \rho(x)}{2 \mu \sqrt{g(x)}}-\frac{i \mu \beta(x)}{2 \sqrt{g(x)}} \\
& a_{22}(x, \mu)=-i \mu b(x)-\frac{g^{\prime}(x)}{4 g(x)}-\frac{d(x) \rho(x)}{\sqrt{g(x)}}+i \frac{q(x) \rho(x)}{2 \mu \sqrt{g(x)}}-\frac{i \mu \beta(x)}{2 \sqrt{g(x)}}
\end{aligned}
$$

For $\mu=s+i \tau$, the entries of the matrix $M_{R}(x, \mu)=\left(M(x, \mu)+M^{*}(x, \mu)\right) / 2$ are:

$$
\begin{aligned}
& a_{11}^{0}(x, \mu)=-\frac{g^{\prime}(x)}{4 g(x)}-\left(\tau \alpha_{1}(x,|\mu|)-\frac{d(x) \rho(x)}{\sqrt{g(x)}}\right), \\
& a_{12}^{0}(x, \mu)=\frac{g^{\prime}(x)}{4 g(x)}+i s \alpha_{2}(x,|\mu|), \\
& a_{21}^{0}(x, \mu)=\frac{g^{\prime}(x)}{4 g(x)}-i s \alpha_{2}(x,|\mu|), \\
& a_{22}^{0}(x, \mu)=-\frac{g^{\prime}(x)}{4 g(x)}+\left(\tau \alpha_{1}(x,|\mu|)-\frac{d(x) \rho(x)}{\sqrt{g(x)}}\right),
\end{aligned}
$$

where

$$
\begin{aligned}
& \alpha_{1}(x,|\mu|)=\frac{\beta(x)}{2 \sqrt{g(x)}}+\frac{q(x) \rho(x)}{2|\mu|^{2} \sqrt{g(x)}}+b(x), \\
& \alpha_{2}(x,|\mu|)=\frac{\beta(x)}{2 \sqrt{g(x)}}-\frac{q(x) \rho(x)}{2|\mu|^{2} \sqrt{g(x)}} .
\end{aligned}
$$


Simple calculations reveal that the eigenvalues of $M_{R}$ are

$$
\begin{aligned}
& \alpha_{ \pm}(x, \mu)=-\frac{g^{\prime}(x)}{4 g(x)} \\
& \quad \pm\left\{\left(\frac{g^{\prime}(x)}{4 g(x)}\right)^{2}+\left(\tau \alpha_{1}(x,|\mu|)-\frac{d(x) \rho(x)}{\sqrt{g(x)}}\right)^{2}+s^{2} \alpha_{2}^{2}(x,|\mu|)\right\}^{\frac{1}{2}} .
\end{aligned}
$$

Note that due to $(2.10)$, for $Y=\left[w_{1}, w_{2}\right]^{T}$ and $\mu \neq 0$, we get

$$
\begin{aligned}
& \|Y(x, \mu)\|^{2}=\left|w_{1}(x, \mu)\right|^{2}+\left|w_{2}(x, \mu)\right|^{2} \\
& \quad=\frac{1}{4|\mu|^{2}}\left(\left|i \mu y(x, \mu)+y^{\prime}(x, \mu) / b(x)\right|^{2}+\left|i \mu y(x, \mu)-y^{\prime}(x, \mu) / b(x)\right|^{2}\right) \\
& \quad=\frac{1}{2|\mu|^{2}}\left(|\mu|^{2}|y(x, \mu)|^{2}+\frac{p(x)\left|y^{\prime}(x, \mu)\right|^{2}}{\eta(x)}\right) .
\end{aligned}
$$

It now follows from the above equation and Proposition 2.2 that for any solution of (1.1) with $\mu \neq 0$ and $\rho$ satisfying (2.7) and (2.8), the following estimations hold

$$
\begin{aligned}
& {\left[|\mu|^{2}|y(0)|^{2}+\frac{p(0)\left|y^{\prime}(0)\right|^{2}}{\eta(0)}\right] \exp \left(2 \int_{0}^{x} \alpha_{-}(t, \tau) d t\right) \leq|\mu|^{2}|y(x)|^{2}+\frac{p(x)\left|y^{\prime}(x)\right|^{2}}{\eta(x)}} \\
& \quad \leq\left[|\mu|^{2}|y(0)|^{2}+\frac{p(0)\left|y^{\prime}(0)\right|^{2}}{\eta(0)}\right] \exp \left(2 \int_{0}^{x} \alpha_{+}(t, \tau) d t\right), \quad x \in[0,1] .
\end{aligned}
$$

On the other hand, for $\alpha_{ \pm}$we have

$$
\left|\alpha_{ \pm}(x, \mu)\right| \leq\left|\frac{g^{\prime}(x)}{2 g(x)}\right|+|\tau|\left|\alpha_{1}(x,|\mu|)\right|+|s|\left|\alpha_{2}(x,|\mu|)\right|+\left|\frac{d(x) \rho(x)}{\sqrt{g(x)}}\right| .
$$

This and the inequality $|s|+|\tau| \leq 2|\mu|$ yields

$$
\left\|\alpha_{ \pm}(x, s+i \tau)\right\|_{L_{1}} \leq \alpha(s, \tau),
$$

where

$$
\alpha(s, \tau)=\frac{\left\|g^{\prime}\right\|_{L_{1}}}{2 m_{g}}+|\tau|\left\|b+\frac{\beta}{2 \sqrt{g}}\right\|_{L_{1}}+|s| \frac{\|\beta\|_{L_{1}}}{2 m_{g}^{1 / 2}}+\frac{M_{\rho}}{m_{g}^{1 / 2}}\left(\frac{\|q\|_{L_{1}}}{|\mu|}+\|d\|_{L_{1}}\right) .
$$

The above relations and (2.14) imply the following inequalities:

$$
\begin{aligned}
& {\left[|\mu|^{2}|y(0)|^{2}+\frac{p(0)\left|y^{\prime}(0)\right|^{2}}{M_{\eta}}\right] \exp (-2 \alpha(s, \tau)) \leq|\mu|^{2}|y(x)|^{2}+\frac{p(x)\left|y^{\prime}(x)\right|^{2}}{m_{\eta}},} \\
& |\mu|^{2}|y(x)|^{2}+\frac{p(x)\left|y^{\prime}(x)\right|^{2}}{M_{\eta}} \leq\left[|\mu|^{2}|y(0)|^{2}+\frac{p(0)\left|y^{\prime}(0)\right|^{2}}{m_{\eta}}\right] \exp (2 \alpha(s, \tau)) .
\end{aligned}
$$


For the second step of the proof assume that $\rho \in L_{\infty}[0,1]$ and $m_{\rho} \leq \rho(x) \leq M_{\rho}$ for a.e. $x \in[0,1]$. It follows from Proposition 2.3 applied with $\delta \in(0,1)$ that we can find a representation

$$
\rho=\rho_{\delta}+\rho_{1, \delta}, \quad \rho_{\delta} \in W_{1}^{1}[0,1], \quad \rho_{1, \delta} \in L_{1}[0,1],
$$

such that

$$
\left\|\rho_{1, \delta}\right\|_{L_{1}} \leq 3 \omega_{1}(\rho, \delta), \quad\left\|\rho_{\delta}^{\prime}\right\|_{L_{1}} \leq \delta^{-1} \omega_{1}(\rho, \delta), \quad m_{\rho} \leq \rho_{\delta}(x) \leq M_{\rho} .
$$

We want to estimate (2.17) in terms of $\rho_{\delta}$ and $\rho_{1, \delta}$ with the use of (2.21). Setting $\eta=\rho_{\delta}$ and $\beta=\rho_{1, \delta}$, we have

$$
b+\frac{\beta}{2 \sqrt{g}}=\frac{1}{2 \sqrt{p}}\left(2 \rho_{\delta}^{1 / 2}+\frac{\rho_{1, \delta}}{\rho_{\delta}^{1 / 2}}\right) .
$$

From (2.20), we obtain

$$
\begin{aligned}
\left\|2 \rho_{\delta}^{1 / 2}+\rho_{1, \delta} / \rho_{\delta}^{1 / 2}\right\|_{L_{1}} & \leq 2\left\|\rho^{1 / 2}\right\|_{L_{1}}+\left\|\left(\rho^{1 / 2}-\rho_{\delta}^{1 / 2}\right)^{2} / \rho_{\delta}^{1 / 2}\right\|_{L_{1}} \\
& \leq 2\left\|\rho^{1 / 2}\right\|_{L_{1}}+\left\|\rho_{1, \delta}\right\|_{L_{1}} / m_{\rho}^{1 / 2} .
\end{aligned}
$$

The expression (2.17) can be estimated for a fixed $\delta \in(0,1)$ by setting $m_{g}=m_{p} m_{\rho}$ and using the above inequality and (2.21):

$$
\begin{aligned}
\alpha_{\delta}(s, \tau) \leq & \frac{M_{p}}{2 m_{g}} \delta^{-1} \omega_{1}(\rho, \delta)+\frac{3}{m_{g}^{1 / 2}}|\mu| \omega_{1}(\rho, \delta)+|\tau| \frac{\left\|\rho^{1 / 2}\right\|_{L_{1}}}{m_{p}^{1 / 2}} \\
& +\frac{M_{\rho}\left\|p^{\prime}\right\|_{L_{1}}}{2 m_{g}}+\frac{M_{\rho}}{m_{g}^{1 / 2}}\left(\frac{\|q\|_{L_{1}}}{|\mu|}+\|d\|_{L_{1}}\right) .
\end{aligned}
$$

Assume $\rho$ is of bounded variation $\mathcal{V}$ on the interval $[0,1]$. It is known that $\omega_{1}(\rho, \delta) \leq \delta \mathcal{V}$. Suppose $|\mu|>1$. Tending with $\delta$ to zero in (2.23), we get

$$
\limsup _{\delta \rightarrow 0} \alpha_{\delta}(s, \tau) \leq \alpha_{0}(|\tau|),
$$

where

$$
\alpha_{0}(|\tau|)=|\tau| \frac{\left\|\rho^{1 / 2}\right\|_{L_{1}}}{m_{p}^{1 / 2}}+\frac{M_{p} \mathcal{V}+M_{\rho}\left\|p^{\prime}\right\|_{L_{1}}}{2 m_{g}}+\frac{M_{\rho}}{m_{g}^{1 / 2}}\left(\|q\|_{L_{1}}+\|d\|_{L_{1}}\right) .
$$

When $\delta$ converges to zero in the representation (2.20) Lemma 2.1 follows from the inequalities (2.18), (2.19), (2.23) and (2.24).

Corollary 2.4. Let $y$ be the solution of the Eq. (1.1) with the initial conditions

$$
y(0)=0, \quad y^{\prime}(0)=1,
$$

then under assumptions of Lemma 2.1 the following inequality is true

$$
\|y(\cdot, \mu)\|_{C} \leq \frac{c}{|\mu|} \exp \left(m_{p}^{-1 / 2}|\tau|\left\|\rho^{1 / 2}\right\|_{L_{1}}\right),
$$

where $\mu=s+i \tau$ and $|\mu|>1$.

Proof. It suffices to consider the inequality (2.2) with the conditions (2.26). 
Remark 2.5. It is possible to obtain estimations analogous to Lemma 2.1 and Corollary 2.4 when $\rho$ satisfies only (1.2) and is not of bounded variation. It suffices to take $\delta=|\mu|^{-1}$ in (2.23) and then the aforementioned inequalities follow only with a factor containing the modulus of continuity and $|\mu|$.

The proof of the main result - Theorem 1.2 - relies also on the following lemma, which is concerned with estimations of solutions, for $\mu$ from the whole complex plane. A part of this will be useful in further applications too.

Lemma 2.6. Let $\rho, p, d$ and $q$ satisfy the assumptions (1.2), (1.3), (1.4), and (1.5). Then for any solution $y=y(x, \mu)$ of the Eq. (1.1), the following inequalities are true for any $\mu \in \mathbb{C}$ :

$$
\begin{aligned}
& \left\|y^{\prime}(\cdot, \mu)\right\|_{C} \leq c\left\{|\mu|^{2}\|y(\cdot, \mu)\|_{L_{1}}+(|\mu|+1)\|y(\cdot, \mu)\|_{C}\right\} \\
& \left\|y^{\prime}(\cdot, \mu)\right\|_{L_{2}}^{2} \leq c\left\{|\mu|^{2}\|y(\cdot, \mu)\|_{L_{1}}+(|\mu|+1)\|y(\cdot, \mu)\|_{C}\right\}\|y(\cdot, \mu)\|_{C} .
\end{aligned}
$$

Moreover, if $f \in \widehat{W}_{2}^{1}[0,1]$, then for any solution $y$ of the Eq. (1.1) the following inequality holds for any $\mu \in \mathbb{C} \backslash\{0\}$ and $x_{1}, x_{2} \in[0,1]$

$$
\begin{aligned}
\left|\int_{x_{1}}^{x_{2}} y(t, \mu) \rho(t) f(t) d t\right| \leq & 3 M_{p}|\mu|^{-2}\left\|y^{\prime}\right\|_{C}\left\|f^{\prime}\right\|_{L_{2}}+|\mu|^{-2} M_{\rho}\|q\|_{L_{1}}\|y\|_{C}\left\|f^{\prime}\right\|_{L_{2}} \\
& +2|\mu|^{-1} M_{\rho}\|d\|_{L_{1}}\|y\|_{C}\left\|f^{\prime}\right\|_{L_{2}} .
\end{aligned}
$$

Proof. Write $y$ instead of $y(\cdot, \mu)$ for convenience. The proof of the estimate (2.28) uses the integral representation for functions from $W_{1}^{1}[0,1]$

$$
g(x)=\int_{0}^{1} S(x, t) g^{\prime}(t) d t+\int_{0}^{1} g(t) d t, \quad g \in W_{1}^{1}[0,1],
$$

where $S(x, t)=t$ for $0 \leq t \leq x$ and $S(x, t)=t-1$ for $x<t \leq 1$. Note that for all $x, t \in[0,1]$ we have $|S(x, t)| \leq 1$. For a solution $y$ of (1.1) set $g=p y^{\prime}$ and $g^{\prime}=\left(2 i \mu d+q-\mu^{2}\right) \rho y$. Using the integral representation (2.31), we obtain

$$
p(x) y^{\prime}(x)=\int_{0}^{1} S(x, t)\left[2 i \mu d(t)+q(t)-\mu^{2}\right] \rho(t) y(t) d t+\int_{0}^{1} p(t) y^{\prime}(t) d t .
$$

Integration of the last term in the equality (2.32) yields

$$
m_{p}\left\|y^{\prime}\right\|_{C} \leq M_{\rho}|\mu|^{2}\|y\|_{L_{1}}+\left\{M_{\rho}\|q\|_{L_{1}}+2 M_{\rho}|\mu|\|d\|_{L_{1}}+2 M_{p}+\left\|p^{\prime}\right\|_{L_{1}}\right\}\|y\|_{C},
$$

hence (2.28) is proved.

We now want to establish (2.29). Multiply (1.1) by $\bar{y}$ and integrate it, so that

$$
\begin{aligned}
\int_{0}^{1} p(t)\left|y^{\prime}(t)\right|^{2} d t= & \int_{0}^{1}\left[\mu^{2}-2 i \mu d(t)-q(t)\right] \rho(t)|y(t)|^{2} d t \\
& +p(1) y^{\prime}(1) \bar{y}(1)-p(0) y^{\prime}(0) \bar{y}(0) .
\end{aligned}
$$


This implies

$$
\begin{aligned}
m_{p}\left\|y^{\prime}\right\|_{L_{2}}^{2} \leq & \left\{M_{\rho}|\mu|^{2}\|y\|_{L_{1}}+\left(M_{\rho}\|q\|_{L_{1}}+2 M_{\rho}|\mu|\|d\|_{L_{1}}\right)\|y\|_{C}\right. \\
& \left.+2 M_{p}\left\|y^{\prime}\right\|_{C}\right\}\|y\|_{C} .
\end{aligned}
$$

Using (2.33), we finally obtain

$$
\begin{aligned}
m_{p}\left\|y^{\prime}\right\|_{L_{2}}^{2} \leq & \|y\|_{C}\left\{|\mu|^{2} M_{\rho}\left(2 m_{p}^{-1} M_{p}+1\right)\|y\|_{L_{1}}+\left(2|\mu| M_{\rho}\|d\|_{L_{1}}\left(2 m_{p}^{-1} M_{p}+1\right)\right.\right. \\
& \left.\left.+2 m_{p}^{-1} M_{p}\left(M_{\rho}\|q\|_{L_{1}}+2 M_{p}+\left\|p^{\prime}\right\|_{L_{1}}\right)+M_{\rho}\|q\|_{L_{1}}\right)\|y\|_{C}\right\},
\end{aligned}
$$

which gives (2.29).

It remains to show (2.30). If $y$ is a solution of (1.1), then

$$
\mu^{2} \rho y=-\left(p y^{\prime}\right)^{\prime}+2 i \mu d \rho y+q \rho y,
$$

and consequently

$$
\begin{aligned}
& \int_{x_{1}}^{x_{2}} \rho(x) y(x) f(x) d x=-\mu^{-2} \int_{x_{1}}^{x_{2}}\left(p(x) y^{\prime}(x)\right)^{\prime} f(x) d x \\
& \quad+2 i \mu^{-1} \int_{x_{1}}^{x_{2}} d(x) \rho(x) y(x) f(x) d x+\mu^{-2} \int_{x_{1}}^{x_{2}} q(x) \rho(x) y(x) f(x) d x .
\end{aligned}
$$

We will now estimate the first integral from (2.34). Due to

$$
\begin{aligned}
\int_{x_{1}}^{x_{2}}\left(p(x) y^{\prime}(x)\right)^{\prime} f(x) d x= & p\left(x_{2}\right) y^{\prime}\left(x_{2}\right) f\left(x_{2}\right)-p\left(x_{1}\right) y^{\prime}\left(x_{1}\right) f\left(x_{1}\right) \\
& -\int_{x_{1}}^{x_{2}} p(x) y^{\prime}(x) f^{\prime}(x) d x,
\end{aligned}
$$

we get

$$
\begin{aligned}
\left|\int_{x_{1}}^{x_{2}}\left(p(x) y^{\prime}(x)\right)^{\prime} f(x) d x\right| & \leq 2 M_{p}\left\|y^{\prime}\right\|_{C}\|f\|_{C}+M_{p}\left\|y^{\prime}\right\|_{C}\left\|f^{\prime}\right\|_{L_{2}} \\
& \leq 3 M_{p}\left\|y^{\prime}\right\|_{C}\left\|f^{\prime}\right\|_{L_{2}},
\end{aligned}
$$

where in the last inequality we used the fact $\|f\|_{C} \leq\left\|f^{\prime}\right\|_{L_{2}}$ for $f \in \widehat{W}_{2}^{1}[0,1]$. Analogously, one can obtain:

$$
\left|\int_{x_{1}}^{x_{2}} d(x) \rho(x) y(x) f(x) d x\right| \leq M_{\rho}\|d\|_{L_{1}}\|y\|_{C}\left\|f^{\prime}\right\|_{L_{2}},
$$




$$
\left|\int_{x_{1}}^{x_{2}} q(x) \rho(x) y(x) f(x) d x\right| \leq M_{\rho}\|q\|_{L_{1}}\|y\|_{C}\left\|f^{\prime}\right\|_{L_{2}} .
$$

Combining the above estimations together concludes the proof.

Corollary 2.7. Let $\rho, p, d$ and $q$ satisfy the assumptions (1.2), (1.3), (1.4), and (1.5). Then for any solution $y=y(x, \mu)$ of the $E q$. (1.1) and $R>0$ there exists a constant $c_{R}$ such that the inequality

$$
\|y\|_{C} \leq c_{R}\|y\|_{L_{1}}
$$

holds for $|\mu| \leq R$.

Proof. From the Nirenberg-Gagliardo inequality (see, e.g., [14, Section 1.2]), for any $y \in W_{1}^{2}[0,1]$ we have

$$
\|y\|_{C} \leq c_{0}\left(\left\|\left(p y^{\prime}\right)^{\prime}\right\|_{L_{1}}+\left\|y^{\prime}\right\|_{C}+\|y\|_{L_{1}}\right)^{1 / 2}\|y\|_{L_{1}}^{1 / 2} .
$$

This implies

$$
2\|y\|_{C}^{2} \leq 2 c_{0}^{2}\left\{\left\|\left(p y^{\prime}\right)^{\prime}\right\|_{L_{1}}+\|y\|_{L_{1}}\right\}\|y\|_{L_{1}}+2 c_{0}^{2}\left\|y^{\prime}\right\|_{C}\|y\|_{L_{1}} .
$$

Note that when $y$ is the solution of (1.1) and $|\mu| \leq R$ we have

$$
\begin{aligned}
& \left\|\left(p y^{\prime}\right)^{\prime}\right\|_{L_{1}}+\|y\|_{L_{1}} \leq\left\|2 i \mu d \rho y+q \rho y-\mu^{2} \rho y\right\|_{L_{1}}+\|y\|_{L_{1}} \\
& \quad \leq\left(M_{\rho}|\mu|^{2}+1\right)\|y\|_{L_{1}}+\left(M_{\rho}\|q\|_{L_{1}}+2 M_{\rho}|\mu|\|d\|_{L_{1}}\right)\|y\|_{C} \\
& \quad \leq c_{1}\|y\|_{L_{1}}+c_{2}\|y\|_{C},
\end{aligned}
$$

where the constants $c_{1}$ and $c_{2}$ depend on $R$. On the other hand, using Cauchy inequality and then (2.28), we obtain for $|\mu| \leq R$ and $\epsilon>0$

$$
\begin{aligned}
2 c_{0}^{2}\left\|y^{\prime}\right\|_{C}\|y\|_{L_{1}} & \leq c_{0}^{2}\left\{\epsilon\left\|y^{\prime}\right\|_{C}^{2}+\epsilon^{-1}\|y\|_{L_{1}}^{2}\right\} \\
& \leq 2 c_{0}^{2} c^{2} \epsilon\left(|\mu|^{4}\|y\|_{L_{1}}^{2}+(|\mu|+1)^{2}\|y\|_{C}^{2}\right)+c_{0}^{2} \epsilon^{-1}\|y\|_{L_{1}}^{2} \\
& \leq 2 c_{0}^{2} c^{2} \epsilon(R+1)^{2}\|y\|_{C}^{2}+c_{0}^{2}\left(2 c^{2} \epsilon R^{4}+\epsilon^{-1}\right)\|y\|_{L_{1}}^{2}
\end{aligned}
$$

Note that we can choose $\epsilon$ such small that $2 c_{0}^{2} c^{2} \epsilon(R+1)^{2} \leq \frac{1}{2}$ and then we have

$$
2 c_{0}^{2}\left\|y^{\prime}\right\|_{C}\|y\|_{L_{1}} \leq \frac{1}{2}\|y\|_{C}^{2}+c_{3}\|y\|_{L_{1}}^{2}
$$

where $c_{3}$ is a constant dependent on $R$ and $\epsilon$. Using (2.37) and (2.38) in (2.36), we obtain

$$
\begin{aligned}
2\|y\|_{C}^{2} & \leq \tilde{c}_{1}\|y\|_{L_{1}}^{2}+2 \tilde{c}_{2}\|y\|_{C}\|y\|_{L_{1}}+\frac{1}{2}\|y\|_{C}^{2}+c_{3}\|y\|_{L_{1}}^{2} \\
& \leq \frac{3}{2}\|y\|_{C}^{2}+\left(\tilde{c}_{1}+\tilde{c}_{2}^{2}+c_{3}\right)\|y\|_{L_{1}}^{2}
\end{aligned}
$$

where constants $\tilde{c}_{1}, \tilde{c}_{2}$ and $c_{3}$ depend only on $R$. This gives

$$
\frac{1}{2}\|y\|_{C}^{2} \leq\left(\tilde{c}_{1}+\tilde{c}_{2}^{2}+c_{3}\right)\|y\|_{L_{1}}^{2} .
$$

We can now prove Theorem 1.2. 
Proof. According to Corollary 2.7 it suffices to establish (1.6) only for $|\mu|>1$. From the inequality (2.2), we obtain

$$
\|y\|_{C}^{2} \leq\left(|y(0)|^{2}+\frac{M_{p}\left|y^{\prime}(0)\right|^{2}}{m_{\rho}|\mu|^{2}}\right) \exp \left(2 \alpha_{0}(|\tau|)\right),
$$

but integrating (2.1) with respect to $x \in[0,1]$ implies

$$
|y(0)|^{2}+\frac{m_{p}\left|y^{\prime}(0)\right|^{2}}{M_{\rho}|\mu|^{2}} \leq\left(\|y\|_{L_{1}}\|y\|_{C}+\frac{M_{p}\left\|y^{\prime}\right\|_{L_{2}}^{2}}{m_{\rho}|\mu|^{2}}\right) \exp \left(2 \alpha_{0}(|\tau|)\right) .
$$

Denote $M=\left(M_{p} M_{\rho}\right) /\left(m_{p} m_{\rho}\right)$ and combine the above inequalities together. The result is

$$
\|y\|_{C}^{2} \leq M\left(\|y\|_{L_{1}}\|y\|_{C}+\frac{M_{p}\left\|y^{\prime}\right\|_{L_{2}}^{2}}{m_{\rho}|\mu|^{2}}\right) \exp \left(4 \alpha_{0}(|\tau|)\right) .
$$

Let $|\operatorname{Im} \mu|=|\tau| \leq r$ for some $r>0$. Using the notation $\widetilde{M}=M_{p} /\left(m_{p} m_{\rho}\right)$ and (2.29) in (2.42) we get

$$
\|y\|_{C} \leq M \exp \left(4 \alpha_{0}(r)\right)\left\{r_{1}\|y\|_{L_{1}}+\left(r_{2}|\mu|^{-1}+r_{3}|\mu|^{-2}\right)\|y\|_{C}\right\},
$$

where:

$$
\begin{aligned}
& r_{1}=1+M\left(2 m_{p}^{-1} M_{p}+1\right), \\
& r_{2}=2 M\|d\|_{L_{1}}\left(2 m_{p}^{-1} M_{p}+1\right), \\
& r_{3}=2 m_{p}^{-1} M_{p}\left(M\|q\|_{L_{1}}+2 \widetilde{M} M_{p}+\widetilde{M}\left\|p^{\prime}\right\|_{L_{1}}\right)+M\|q\|_{L_{1}} .
\end{aligned}
$$

Take $r_{0}=2 M\left(r_{2}+r_{3}\right) \exp \left(4 \alpha_{0}(r)\right)$. Then for $|\mu|>\max \left\{r_{0}, 1\right\}$ we obtain (1.6) with the constant $c=2 M r_{1} \exp \left(4 \alpha_{0}(r)\right)$.

\section{Applications}

The focus of this section is on the application of results of Sect. 2 to the problem of an inhomogeneous, damped string of length one. We assume that the string has left end fixed and right one moving with damping. The density of the string will be denoted as $\rho$. The string is characterized by the modulus of elasticity, which is called $p$, while the function $q$ is the potential. We also claim presence of viscous damping with the friction coefficient $2 d$.

Denote the vertical position of the string in time $t \in[0, \infty)$ on the interval $[0,1]$ by $v=v(x, t)$. Then small vibrations are described by the wave equation

$$
v_{t t}(x, t)-\rho(x)^{-1}\left(p(x) v_{x}(x, t)\right)_{x}+2 d(x) v_{t}(x, t)+q(x) v(x, t)=0,
$$

with the boundary conditions:

$$
v(0, t)=0, \quad v_{x}(1, t)+h v_{t}(1, t)=0,
$$

and the initial conditions:

$$
v(x, 0)=v_{0}(x), \quad v_{t}(x, 0)=v_{1}(x) .
$$


The functions $v_{0}$ and $v_{1}$ are initial position and velocity, respectively. Parameter $h \in \mathbb{C}$ in (3.2) is allowed to be complex, for then we can deal with a broader class of physical phenomena related with the string equation (see, e.g., instance [20] and the references therein). This is why we regard $v:[0,1] \times[0, \infty) \rightarrow \mathbb{C}$ as a complex-valued function.

In this section we assume:

$$
\rho \in L_{\infty}[0,1], \quad 0<m_{\rho} \leq \rho(x) \leq M_{\rho} \text { for a.e. } x \in[0,1],
$$

and is of bounded variation. The remaining functions are real valued and satisfy

$$
\begin{aligned}
& p \in W_{1}^{1}[0,1], \quad 0<m_{p} \leq p(x) \leq M_{p}<\infty, \quad x \in[0,1] . \\
& d \in L_{1}[0,1], \quad d \geq 0, \\
& q \in L_{1}[0,1], \quad q \geq 0 .
\end{aligned}
$$

Note that the assumptions in this section allows to use the results of the previous section.

In what follows we understand as $\widehat{L}_{2}[0,1]$ the weighted $L_{2}[0,1]$ space with the scalar product

$$
\left\langle v_{1}, v_{2}\right\rangle_{\widehat{L}_{2}}=\int_{0}^{1} \rho(x) v_{1}(x) \overline{v_{2}}(x) d x,
$$

while $\widehat{W}_{2}^{1}[0,1]=\left\{y \in W_{2}^{1}[0,1] ; y(0)=0\right\}$ is equipped with

$$
\left\langle u_{1}, u_{2}\right\rangle_{\widehat{W}_{2}^{1}}=\int_{0}^{1}\left(p(x) u_{1}^{\prime}(x) \bar{u}_{2}^{\prime}(x)+q(x) \rho(x) u_{1}(x) \bar{u}_{2}(x)\right) d x,
$$

which, in view of assumptions on $\rho, p$ and $q$, induces the norm equivalent to the classical one:

$$
\|y\|_{\widehat{W}_{2}^{1}}^{2}=\int_{0}^{1}\left(\left|y^{\prime}(x)\right|^{2}+|y(x)|^{2}\right) d x .
$$

The problem (3.1)-(3.3) can be transformed into an abstract Cauchy problem

$$
\begin{aligned}
V^{\prime}(t) & =B_{h} V(t), \quad t>0, \\
V(0) & =\left[v_{0}, v_{1}\right]^{T},
\end{aligned}
$$

in the Hilbert space

$$
H=\widehat{W}_{2}^{1}[0,1] \oplus \widehat{L}_{2}[0,1] .
$$

It suffices to take $V(t)=\left[v(\cdot, t), v_{t}(\cdot, t)\right]^{T}$ and the linear operator $B_{h}$ : $\mathcal{D}\left(B_{h}\right) \rightarrow H$ defined by

$$
B_{h}=\left[\begin{array}{cc}
0 & I \\
\rho^{-1} \frac{d}{d x}\left(p \frac{d}{d x} \cdot\right) & -q-2 d
\end{array}\right],
$$


where $I$ is the identity operator on $\widehat{W}_{2}^{1}[0,1]$. The domain is

$$
\begin{gathered}
\mathcal{D}\left(B_{h}\right)=\{(u, v) \\
u W_{1}^{2}[0,1] \oplus \widehat{W}_{2}^{1}[0,1] ; \rho^{-1}\left(p u^{\prime}\right)^{\prime}-q u-2 d v \in L_{2}[0,1], \\
\left.u(0)=0, u^{\prime}(1)+h v(1)=0\right\} .
\end{gathered}
$$

The first component $u$ of the domain is $W_{1}^{2}[0,1]$ since it should be coherent with Definition 1.1.

Our main aim in this section is to prove that $B_{h}$ is a generator of the exponentially stable $C_{0}$-semigroup $e^{B_{h} t}, t \geq 0$, of contractions. We will follow the methods used in [19], where the problem (3.1)-(3.3) was considered with $p=1, d=0$ and $q=0$. Particular cases of the problem of a damped string and its energy with different types of boundary conditions were investigated for instance in $[1,2,12]$, but in each case some of coefficients were zero. The main advantage of our approach is the presence of nontrivial functions $p, d$, $q$ and weak assumptions on them. The fact that $\rho$ is of bounded variation is also important, since usually it is claimed that $\rho$ is differentiable or an element of a Sobolev space. Extensive literature on the problem of a damped string can be found in [10]. For some applications of this problem see [20,21]. An interesting approach to the problem for homogeneous string can be found in $[5,16,17]$, where a damping which is indefinite occurs.

The norm in the space $H$ was chosen in such a way that the square of the norm of a solution of the problem (3.11)-(3.12) is the physical energy of the string. Therefore $H$ is called the energy space. In this norm the inequality $\operatorname{Re}\left\langle B_{h} x, x\right\rangle_{H} \leq 0$ holds for $x \in \mathcal{D}\left(B_{h}\right)$, hence $B_{h}$ is dissipative. One can show that $B_{h}$ has no dissipative extensions, thus $B_{h}$ is maximal dissipative. This property is important in theorems of $C_{0}$-semigroup generation.

When $B_{h}$ is the generator of an exponentially stable $C_{0}$-semigroup of contractions, the solution of the problem (3.11)-(3.12) exists and converges exponentially to zero with respect to the energy norm. Consequently, physical energy of the string decays exponentially in time.

We want to investigate spectral properties of the operator $B_{h}$ given by the expressions (3.13)-(3.14). In particular, we will establish that $B_{h}$ is closed, densely defined and invertible. For convenience we introduce an operator $A_{h}$ defined on $D\left(A_{h}\right)=D\left(B_{h}\right)$ by

$$
A_{h}=-i\left[\begin{array}{cc}
0 & I \\
\rho^{-1} \frac{d}{d x}\left(p \frac{d}{d x} \cdot\right) & -q-2 d
\end{array}\right],
$$

so that $B_{h}=i A_{h}$. The following properties are true for the operator $A_{h}$.

Proposition 3.1. Under assumptions (3.4)-(3.7) the spectrum $\sigma\left(A_{h}\right)$ consists of at most a countable number of nonzero points with the accumulation point at infinity. Moreover, every $\mu \in \sigma\left(A_{h}\right)$ is an eigenvalue. The operator $A_{h}$ is densely defined and closed.

Proof. The resolvent $\left(A_{h}-\mu I\right)^{-1}$ of the operator $A_{h}$ is defined by the equation

$$
\left(A_{h}-\mu I\right)(u, v)=(f, g)
$$


where $(u, v) \in \mathcal{D}\left(A_{h}\right)$ and $(f, g) \in H$. This can be transformed into the boundary value problem

$$
\begin{gathered}
l_{\mu}(u)=F(x, \mu), \\
u(0)=0, \quad U[u](\mu):=u^{\prime}(1)+i \mu h u(1)=-i h f(1),
\end{gathered}
$$

where

$$
F(x, \mu)=\rho(x)[i(g(x)+2 d(x) f(x))-\mu f(x)] \in L_{1}[0,1],
$$

and $v$ is expressed by the formula

$$
v(x)=i(f(x)+\mu u(x)) .
$$

The Eq. (3.17) is an inhomogeneous case of the Sturm-Liouville equation (1.1) from Sect. 2. We want to express the solution of (3.17)-(3.17) by solutions $y_{1}=y_{1}(x, \mu), y_{2}=y_{2}(x, \mu)$ of $l_{\mu}(y)=0$ with initial conditions

$$
y_{1}(0, \mu)=0, \quad y_{1}^{\prime}(0, \mu)=1, \quad y_{2}(0, \mu)=-1, \quad y_{2}^{\prime}(0, \mu)=0 .
$$

The problem $l_{\mu}\left(y_{1}\right)=0$ with appropriate initial conditions can be transformed into a first order system

$$
\begin{aligned}
Z^{\prime} & =R(\mu) Z, \\
Z(0) & =[0, p(1)]^{T}
\end{aligned}
$$

where $Z=\left[y_{1}, z\right]^{T}, z=p y_{1}^{\prime}$ and $R(\mu)$ is given by

$$
R(\mu)=\left[\begin{array}{cc}
0 & 1 / p \\
\left(q+2 i \mu d-\mu^{2}\right) \rho & 0
\end{array}\right] .
$$

Proceeding in the same way as in [24, Theorem 1.2.1], one can prove the existence of unique solution of the above system which is analytic with respect to $\mu$. Therefore there exists the solution $y_{1}=y_{1}(x, \mu)$ of the equation $l_{\mu}\left(y_{1}\right)=0$ such that $y_{1}$ is analytic with respect to $\mu$. Analogous result can be obtained for $y_{2}$. Note that in view of (3.21) we have the following identity for Wronskian:

$$
W\left(y_{1}, y_{2}, \mu\right)=p(0)\left(y_{1}(0, \mu) y_{2}^{\prime}(0, \mu)-y_{1}^{\prime}(0, \mu) y_{2}(0, \mu)\right)=p(0) .
$$

Then the particular solution $y_{0}=y_{0}(x, \mu) \in \widehat{W}_{1}^{2}[0,1]$ of the inhomogeneous Eq. (3.17) is given by the formula

$$
\begin{aligned}
y_{0}(x, \mu)= & \frac{y_{2}(x, \mu)}{p(0)} \int_{0}^{x} y_{1}(t, \mu) F(t, \mu) d t \\
& +\frac{y_{1}(x, \mu)}{p(0)} \int_{x}^{1} y_{2}(t, \mu) F(t, \mu) d t .
\end{aligned}
$$

Let $u(x, \mu)=C y_{1}(x, \mu)+y_{0}(x, \mu)$. By using boundary conditions (3.18), we obtain

$$
u(x, \mu)=-\frac{i h f(1)+U\left[y_{0}\right](\mu)}{U\left[y_{1}\right](\mu)} y_{1}(x, \mu)+y_{0}(x, \mu) .
$$


Note that $u \in \widehat{W}_{1}^{2}[0,1]$. Consider the operator $Q: H \rightarrow \mathcal{D}\left(A_{h}\right)$ which acts as follows $Q(f, g)=(u, v)$ where $u$ is given by (3.24) and $v$ by (3.20). We will prove that this operator is bounded on $H$ whenever the expression (3.24) is well defined i.e. $U\left[y_{1}\right](\mu) \neq 0$. The expression (3.23) for $y_{0}$ can be considered as an operator which assign $L_{1}[0,1] \ni F \mapsto y_{0} \in \widehat{W}_{2}^{1}[0,1]$. In view of the formula for the derivative

$$
\begin{aligned}
y_{0}^{\prime}(x, \mu)= & \frac{y_{2}^{\prime}(x, \mu)}{p(0)} \int_{0}^{x} y_{1}(t, \mu) F(t, \mu) d t \\
& +\frac{y_{1}^{\prime}(x, \mu)}{p(0)} \int_{x}^{1} y_{2}(t, \mu) F(t, \mu) d t,
\end{aligned}
$$

we see that it is the bounded operator acting from $L_{1}[0,1]$ to $\widehat{W}_{2}^{1}[0,1]$. If we consider the expression (3.19) as an operator which assign $(f, g) \mapsto F(x, \mu)$ then one can show that it is the bounded operator from $H$ to $L_{1}[0,1]$. This gives the boundedness of $y_{0}: H \rightarrow \widehat{W}_{2}^{1}[0,1]$. Note that

$$
\left|i h f(1)+U\left[y_{0}\right](\mu)\right| \leq c\left(\|f\|_{\widehat{W}_{2}^{1}}+\|g\|_{\widehat{L}_{2}}\right) .
$$

Consequently, the first part of (3.24) is a one-dimensional compact operator acting on $H \rightarrow \widehat{W}_{2}^{1}[0,1]$. Proceeding in the similar way as for $u$, one can show that $v$ considered as an operator $H \rightarrow \widehat{L}_{2}[0,1]$ defined by $(3.20)$ is bounded. To sum up, the operator $Q$ is the resolvent of $A_{h}$ if $U\left[y_{1}\right](\mu) \neq 0$. According to (3.24) the resolvent exists for all $\mu$ which does not coincide with the roots of the analytic function $U\left[y_{1}\right](\mu)$. In particular, one can show that $U\left[y_{1}\right](0) \neq 0$, hence this is a nonzero function. As a consequence the spectrum of $A_{h}$ has the form

$$
\sigma\left(A_{h}\right)=\left\{\mu \in \mathbb{C}: U\left[y_{1}\right](\mu)=0\right\},
$$

thus it consists of at most a countable number of nonzero points with the accumulation point at infinity. It follows that $A_{h}$ is closed. Moreover, simple calculations reveal that the eigenfunction corresponding to an eigenvalue $\mu$ is given by $\left[y_{1}(\cdot, \mu), i \mu y_{1}(\cdot, \mu)\right]^{T}$.

We now want to provide that $A_{h}$ is densely defined. Note that for all $(u, v) \in \mathcal{D}\left(A_{h}\right)$ and $(f, g) \in H$ we have

$$
\begin{aligned}
\langle(u, v),(f, g)\rangle_{H}= & -\int_{0}^{1} l_{0}(u)(x) \overline{f(x)} d x+\int_{0}^{1} \rho(x) v(x) \overline{g(x)} d x \\
& +p(1) u^{\prime}(1) \overline{f(1)} .
\end{aligned}
$$

Suppose that there exists $(f, g) \in H$ such that for all $(u, v) \in \mathcal{D}\left(A_{h}\right)$ we have $\langle(u, v),(f, g)\rangle_{H}=0$. If $u=0$ then (3.27) implies that for all $v \in \widehat{W}_{2}^{1}[0,1]$ the following identity is true:

$$
\int_{0}^{1} \rho(x) v(x) \overline{g(x)} d x=0,
$$


thus $g=0$. We will show that $f=0$. If $v=0$, then again by (3.27) for all $(u, 0) \in \mathcal{D}\left(A_{h}\right)$ we have

$$
-\int_{0}^{1} l_{0}(u)(x) \overline{f(x)} d x+p(1) u^{\prime}(1) \overline{f(1)}=0 .
$$

On the other hand the existence of $A_{h}^{-1}$ yields that for all $(\widehat{f}, \widehat{g}) \in H$ the equation $A_{h}(u, v)=(\widehat{f}, \widehat{g})$ has the unique solution $(u, v) \in \mathcal{D}\left(A_{h}\right)$. Equivalently $(u, v)$ confirms $(3.17)-(3.20)$ for $\mu=0$. Set $\widehat{f}=0$ and $\widehat{g}=f$. In view of (3.20) we get $v=0$ and there exists $u$ such that $l_{0}(u)=i \rho f$ with conditions $u(0)=0$ and $u^{\prime}(1)=0$. Using this in (3.28), we obtain

$$
\int_{0}^{1} \rho(x)|f(x)|^{2} d x=0,
$$

hence $f=0$.

Obviously, all of those results are also true for $B_{h}$. Other properties of the operator $B_{h}$ such as base properties were studied in $[22,23]$, but with stronger assumptions on $p, d, q$ and $\rho$.

Exponential stability of $B_{h}$ can be established as a consequence of the Gearhart Theorem [4, Chapter V,Theorem 1.11].

Proposition 3.2. [19, Proposition 3.2] Let B be a linear operator acting on the Hilbert space $H$ which generates a uniformly bounded $C_{0}$-semigroup of operators $T(t), t \geq 0$. Suppose that there exists the resolvent on the imaginary axis which is uniformly bounded, i.e.,

$$
\left\|(B-i \tau I)^{-1}\right\| \leq r, \quad \tau \in \mathbb{R}, \quad r>0 .
$$

Then the semigroup $T(t)$ is exponentially stable. Moreover, for any $0<\delta<r^{-1}$ there exists a constant $M_{\delta}>0$ such that

$$
\|T(t)\| \leq M_{\delta} e^{-\delta t}, \quad t \geq 0 .
$$

According to Proposition 3.2, first we need to show that $B_{h}$ is the generator of a $C_{0}$-semigroup of contractions. Then we should establish the existence of the resolvent of the operator $B_{h}$ on the imaginary axis and find its form, which is easy to estimate. This is where the inequalities for the solutions of the Sturm-Liouville equation are needed.

It is well-known that a densely defined, maximal dissipative operator generates a contraction $C_{0}$-semigroup (see [4, Chapter II, Theorem 3.15]). We will use this fact to prove our first result.

Theorem 3.3. Let $p, d$ and $q$ satisfy assumptions (3.5), (3.6) and (3.7). Moreover, let $\rho$ be as in (3.4) and $\operatorname{Re} h \geq 0$. Then the operator $B_{h}$ generates a $C_{0}$-semigroup of contractions in the space $H=\widehat{W}_{2}^{1}[0,1] \oplus \widehat{L}_{2}[0,1]$.

Proof. We mentioned before that $B_{h}$ is densely defined, thus it remains to prove $B_{h}$ is maximal dissipative. Take $w=(u, v) \in \mathcal{D}\left(B_{h}\right)$. After integration, 
we have

$$
\begin{aligned}
\left\langle B_{h} w, w\right\rangle_{H} & =\left\langle\left(v, 1 / \rho\left(p u^{\prime}\right)^{\prime}-q u-2 d v\right),(u, v)\right\rangle_{H} \\
& =\langle v, u\rangle_{\widehat{W}_{2}^{1}}-\langle u, v\rangle_{\widehat{W}_{2}^{1}}+p(1) u^{\prime}(1) \overline{v(1)}-2\|\sqrt{d} v\|_{\widehat{L}_{2}} \\
& =2 i \operatorname{Im}\langle v, u\rangle_{\widehat{W}_{2}^{1}}-h p(1)|v(1)|^{2}-2\|\sqrt{d} v\|_{\widehat{L}_{2}},
\end{aligned}
$$

hence

$$
\operatorname{Re}\left\langle B_{h} w, w\right\rangle_{H}=-\operatorname{Re} h p(1)|v(1)|^{2}-2\|\sqrt{d} v\|_{\widehat{L}_{2}} .
$$

Summarizing, the operator $B_{h}$ is dissipative whenever $\operatorname{Re} h \geq 0$. The inverse of $B_{h}$ is bounded and zero is an element of the resolvent set $\rho\left(B_{h}\right)$. This set is open, thus there exists $\mu>0$ in $\rho\left(B_{h}\right)$ which yields gives maximal dissipativity of $B_{h}$.

Remark 3.4. In what follows we exclude the case $\operatorname{Re} h=0$ and $d=0$. In this case the operator $B_{h}$ is skew-adjoint and there is no exponential stability of $e^{B_{h} t}$.

According to Theorem 3.3 what is left to show is the existence of the resolvent of $B_{h}$ on the imaginary axis and its estimation. Instead of this we will establish that the spectrum of $A_{h}$ is separated from the real axis. Additionally, we provide a lower bound for the imaginary part of eigenvalues of $A_{h}$.

Lemma 3.5. Let the functions $p, d$, $q$ be as in (3.5), (3.6), (3.7). Furthermore, let $\rho$ be of bounded variation $\mathcal{V}$ and satisfying (3.4). Whenever $\operatorname{Re} h>0$, then there exists a constant $c>0$ such that for any eigenvalue $\mu$ of the operator $A_{h}$, the following inequality holds

$$
\operatorname{Im} \mu \geq c>0 .
$$

Proof. It was shown that if $\operatorname{Re} h>0$ then the operator $B_{h}$ is dissipative and $\operatorname{Im}\left\langle A_{h} w, w\right\rangle_{H} \geq 0$, hence for $\mu \in \sigma\left(A_{h}\right)$ we have $\operatorname{Im} \mu \geq 0$. We will show that eigenvalues are separated from the real axis. If $\mu$ is an eigenvalue of $A_{h}$ with eigenfunction $w=(u, v)$, then from (3.15) we have $v=i \mu u$, where $u$ is a solution of

$$
l_{\mu}(u)=0, \quad x \in[0,1],
$$

with boundary conditions

$$
u(0)=0, \quad U[u](\mu)=u^{\prime}(1)+i \mu h u(1)=0 .
$$

Multiplying (3.32) by $\bar{u}$ and integrating by parts gives

$$
\begin{aligned}
0= & -i \mu h p(1)|u(1)|^{2}-\int_{0}^{1} p(x)\left|u^{\prime}(x)\right|^{2} d x+\mu^{2} \int_{0}^{1} \rho(x)|u(x)|^{2} d x \\
& -2 i \mu \int_{0}^{1} d(x) \rho(x)|u(x)|^{2} d x-\int_{0}^{1} q(x) \rho(x)|u(x)|^{2} d x
\end{aligned}
$$


Dividing (3.34) by $\mu=s+i \tau$, we have

$$
\begin{aligned}
i h p(1)|u(1)|^{2}= & \mu \int_{0}^{1} \rho(x)|u(x)|^{2} d x-2 i \int_{0}^{1} d(x) \rho(x)|u(x)|^{2} d x \\
& -\frac{1}{\mu} \int_{0}^{1} q(x) \rho(x)|u(x)|^{2} d x-\frac{1}{\mu} \int_{0}^{1} p(x)\left|u^{\prime}(x)\right|^{2} d x .
\end{aligned}
$$

Denote $\mu=s+i \tau$ and $h=a+i b$. Taking the imaginary part of the above equation implies

$$
\begin{aligned}
a p(1)|u(1)|^{2}= & \tau \int_{0}^{1} \rho(x)|u(x)|^{2} d x-2 \int_{0}^{1} d(x) \rho(x)|u(x)|^{2} d x \\
& +\frac{\tau}{|\mu|^{2}} \int_{0}^{1} q(x) \rho(x)|u(x)|^{2} d x+\frac{\tau}{|\mu|^{2}} \int_{0}^{1} p(x)\left|u^{\prime}(x)\right|^{2} d x .
\end{aligned}
$$

Assumptions on $\rho, p, d$ and $q$ give us the inequality

$$
a m_{p}|u(1)|^{2} \leq \tau\left(M_{\rho}\|u\|_{L_{2}}^{2}+\frac{M_{p}\left\|u^{\prime}\right\|_{L_{2}}^{2}+M_{\rho}\|q\|_{L_{1}}\|u\|_{C}^{2}}{|\mu|^{2}}\right) .
$$

Consequently, we obtain

$$
a m_{p}|u(1)|^{2} \leq \tau M_{0}\left(M_{\rho}\|u\|_{L_{2}}^{2}+\frac{m_{p}\left\|u^{\prime}\right\|_{L_{2}}^{2}}{|\mu|^{2}}\right),
$$

where $M_{0}=\max \left\{1, m_{p}^{-1}\left(M_{p}+M_{\rho}\|q\|_{L_{1}}\right)\right\}$. Our aim now is to establish the lower bound of $|u(1)|$. Lemma 2.1 and conditions (3.33) yield

$$
\begin{aligned}
|\mu|^{2}|u(1)|^{2}\left(1+M_{p}|h|^{2} m_{\rho}^{-1}\right) & =|\mu|^{2}|u(1)|^{2}+M_{p}\left|u^{\prime}(1)\right|^{2} / m_{\rho} \geq \\
& \geq\left(|\mu|^{2}|u(0)|^{2}+m_{p}\left|u^{\prime}(0)\right|^{2} / M_{\rho}\right) e^{-2 \alpha_{0}(\tau)} \\
& \geq \frac{m_{\rho}}{M_{\rho}^{2}}|\mu|^{2}\left(M_{\rho}|u(x)|^{2}+\frac{m_{p}\left|u^{\prime}(x)\right|^{2}}{|\mu|^{2}}\right) e^{-4 \alpha_{0}(\tau)} .
\end{aligned}
$$

After integration, we get

$$
|u(1)|^{2} \geq \frac{m_{\rho}^{2}}{M_{\rho}^{2}\left(m_{\rho}+M_{p}|h|^{2}\right)}\left(M_{\rho}\|u\|_{L_{2}}^{2}+\frac{m_{p}\left\|u^{\prime}\right\|_{L_{2}}^{2}}{|\mu|^{2}}\right) e^{-4 \alpha_{0}(\tau)} .
$$

Denote

$$
\alpha:=\frac{M_{p} \mathcal{V}+M_{\rho}\left\|p^{\prime}\right\|_{L_{1}}}{2 m_{g}}+\frac{M_{\rho}}{m_{g}^{1 / 2}}\left(\|q\|_{L_{1}}+\|d\|_{L_{1}}\right) .
$$

Combining (3.36), (3.37) and the inequality $e^{-x} \geq 1-x$ for $x \geq 0$, we obtain

$$
\begin{aligned}
\tau & \geq \frac{a m_{p} m_{\rho}^{2}}{M_{0} M_{\rho}^{2}\left(m_{\rho}+M_{p}|h|^{2}\right)} \exp (-4 \alpha) \exp \left(-4 \tau\left\|\rho^{1 / 2}\right\|_{L_{1}} / m_{p}^{1 / 2}\right) \\
& \geq \frac{a m_{p} m_{\rho}^{2}}{M_{0} M_{\rho}^{2}\left(m_{\rho}+M_{p}|h|^{2}\right)} \exp (-4 \alpha)\left(1-4 \tau\left\|\rho^{1 / 2}\right\|_{L_{1}} / m_{p}^{1 / 2}\right) .
\end{aligned}
$$


This finally gives

$$
\tau \geq c_{0}\left(1+4 c_{0}\left\|\rho^{1 / 2}\right\|_{L_{1}} / m_{p}^{1 / 2}\right)^{-1}>0,
$$

with the constant $c_{0}=\frac{a m_{p} m_{\rho}^{2}}{M_{0} M_{\rho}^{2}\left(m_{\rho}+M_{p}|h|^{2}\right)} \exp (-4 \alpha)$.

We can now state and prove the second theorem about generation.

Theorem 3.6. Let $\operatorname{Re} h>0$ and $p, d, q$ satisfy assumptions (3.5), (3.6), (3.7). Moreover, let $\rho$ be as in (3.4) and of bounded variation. Then the operator $B_{h}$ generates an exponentially stable $C_{0}$-semigroup in the space $H=\widehat{W}_{2}^{1}[0,1] \oplus \widehat{L}_{2}[0,1]$.

Proof. By Proposition 3.2, we need to provide

$$
\left\|\left(A_{h}-s I\right)^{-1}\right\| \leq r, \quad s \in \mathbb{R}, \quad r>0 .
$$

Due to the analyticity of the resolvent it is enough to prove (3.39) only for $|s|>1$.

For an arbitrary $w=(f, g) \in H$ and $s \in \mathbb{R},(3.20)$ implies the following estimation of the resolvent

$$
\begin{aligned}
& \left\|\left(A_{h}-s I\right)^{-1} w\right\|_{H}^{2} \\
& \quad=\int_{0}^{1} p(x)\left|u^{\prime}(x, s)\right|^{2} d x+\int_{0}^{1} q(x) \rho(x)|u(x, s)|^{2} d x+\int_{0}^{1} \rho(x)|v(x, s)|^{2} d x \\
& \quad \leq M_{p}\left\|u^{\prime}(\cdot, s)\right\|_{L_{2}}^{2}+M_{\rho}\|q\|_{L_{1}}\|u(\cdot, s)\|_{C}^{2}+2 M_{\rho} s^{2}\|u(\cdot, s)\|_{L_{2}}^{2}+2 M_{\rho}\|f\|_{L_{2}}^{2} .
\end{aligned}
$$

According to (3.40), it suffices to establish the following estimations for $|s|>1$ :

$$
\|u(\cdot, s)\|_{L_{2}} \leq\|u(\cdot, s)\|_{C} \leq c|s|^{-1}\|w\|_{H}, \quad\left\|u^{\prime}(\cdot, s)\right\|_{L_{2}} \leq c\|w\|_{H} .
$$

We will estimate the expressions from (3.24) one by one. We need a lower bound of $\left|U\left[y_{1}\right](s)\right|$. Write $h=a+i b$ and recall that $a>0$. When $s \in \mathbb{R}$, the functions $y_{1}$ and $y_{2}$ are real-valued and this gives

$$
\left|U\left[y_{1}\right](s)\right|^{2}=\left(y_{1}^{\prime}(1, s)\right)^{2}+s^{2} a^{2} y_{1}^{2}(1, s)+s^{2} b^{2} y_{1}^{2}(1, s)-2 b s y_{1}(1, s) y_{1}^{\prime}(1, s) .
$$

Recall the numerical Cauchy inequality $2 x y \leq \xi x^{2}+\frac{y^{2}}{\xi}$ for $x, y \in \mathbb{R}$ and $\xi>0$. When $b \neq 0$, we can use this in the last term of (3.41), and this implies

$$
\left|U\left[y_{1}\right](s)\right|^{2} \geq\left(y_{1}^{\prime}(1, s)\right)^{2}\left(1-\xi^{-1}\right)+y_{1}^{2}(1, s) s^{2}\left(a^{2}+b^{2}-b^{2} \xi\right) .
$$

Since $b \neq 0$ and $a>0$, there is always $\xi$ such that $1<\xi<1+\frac{a^{2}}{b^{2}}$, hence the minimum $k=\min \left\{m_{\rho}\left(1-\xi^{-1}\right) / M_{p}, a^{2}+b^{2}-b^{2} \xi\right\}$ is positive. Simple calculations reveal that 


$$
\begin{aligned}
k & =\frac{1}{2}\left(|h|^{2}+m_{\rho} / M_{p}-\sqrt{\left(m_{\rho} / M_{p}-|h|^{2}\right)^{2}+4 m_{\rho} / M_{p} b^{2}}\right) \\
& \geq k_{0}=\frac{a^{2} m_{\rho} / M_{p}}{|h|^{2}+m_{\rho} / M_{p}}>0 .
\end{aligned}
$$

If $b=0$, then $k_{0}=\min \left\{m_{\rho} / M_{p}, a^{2}\right\}$. Thanks to conditions (3.21) and Lemma 2.1 , we obtain

$$
\begin{aligned}
\left|U\left[y_{1}\right](s)\right|^{2} & \geq k_{0}\left(s^{2} y_{1}^{2}(1, s)+\frac{M_{p}\left(y_{1}^{\prime}(1, s)\right)^{2}}{m_{\rho}}\right) \\
& \geq k_{0} C_{1}^{-1}\left(s^{2} y_{1}^{2}(0, s)+\frac{m_{p}\left(y_{1}^{\prime}(0, s)\right)^{2}}{M_{\rho}}\right) \\
& =k_{0} m_{p} C_{1}^{-1} M_{\rho}^{-1}>0
\end{aligned}
$$

where $C_{1}=\exp \left(2 \alpha_{0}(0)\right)$. Consequently,

$$
\left|U\left[y_{1}\right](s)\right|^{-1} \leq c_{0}=\left(k_{0}^{-1} m_{p}^{-1} C_{1} M_{\rho}\right)^{\frac{1}{2}}
$$

The next step is to estimate the norms of $y_{1}$ and $y_{2}$ in $C[0,1]$ and also of their derivatives. Define an even real function $\mathbb{R} \ni s \mapsto z(s)$ by

$$
z^{2}(s)=s^{2}\left\|y_{1}(\cdot, s)\right\|_{C}^{2}+\left\|y_{1}^{\prime}(\cdot, s)\right\|_{C}^{2}+\left\|y_{2}(\cdot, s)\right\|_{C}^{2}+s^{-2}\left\|y_{2}^{\prime}(\cdot, s)\right\|_{C}^{2} .
$$

If we show that $z$ is bounded by some constant $c_{1}$, then it will follow immediately from its definition that

$$
\begin{aligned}
\left\|y_{1}(\cdot, s)\right\|_{L_{2}} & \leq\left\|y_{1}(\cdot, s)\right\|_{C} \leq z(s)|s|^{-1} \leq c_{1}|s|^{-1} \\
\left\|y_{1}^{\prime}(\cdot, s)\right\|_{L_{2}} & \leq\left\|y_{1}^{\prime}(\cdot, s)\right\|_{C} \leq z(s) \leq c_{1} \\
\left\|y_{2}(\cdot, s)\right\|_{L_{2}} & \leq\left\|y_{2}(\cdot, s)\right\|_{C} \leq z(s) \leq c_{1} \\
\left\|y_{2}^{\prime}(\cdot, s)\right\|_{L_{2}} & \leq\left\|y_{2}^{\prime}(\cdot, s)\right\|_{C} \leq z(s)|s| \leq c_{1}|s|
\end{aligned}
$$

Denote $M_{1}=\max \left\{1, M_{\rho} / m_{p}\right\}$. Again by Lemma 2.1 and (3.21), we have

$$
\begin{aligned}
& M_{1}\left(1+m_{\rho}^{-1} M_{p}\right) C_{1} \\
& =M_{1} C_{1}\left(s^{2}\left|y_{1}(0, s)\right|^{2}+m_{\rho}^{-1} M_{p}\left|y_{1}^{\prime}(0, s)\right|^{2}+\left|y_{2}(0, s)\right|^{2}\right. \\
& \left.\quad+s^{-2} m_{\rho}^{-1} M_{p}\left|y_{2}^{\prime}(0, s)\right|^{2}\right) \\
& \geq M_{1}\left(s^{2}\left|y_{1}(x, s)\right|^{2}+M_{\rho}^{-1} m_{p}\left|y_{1}^{\prime}(x, s)\right|^{2}+\left|y_{2}(x, s)\right|^{2}\right. \\
& \left.\quad+s^{-2} M_{\rho}^{-1} m_{p}\left|y_{2}^{\prime}(x, s)\right|^{2}\right) \\
& \geq s^{2}\left|y_{1}(x, s)\right|^{2}+\left|y_{1}^{\prime}(x, s)\right|^{2}+\left|y_{2}(x, s)\right|^{2}+s^{-2}\left|y_{2}^{\prime}(x, s)\right|^{2},
\end{aligned}
$$

thus

$$
z(s) \leq c_{1}=2\left(M_{1}\left(1+m_{\rho}^{-1} M_{p}\right) C_{1}\right)^{\frac{1}{2}}
$$


We can now estimate $\left|U\left[y_{0}\right](s)\right|$. By (3.43), we obtain

$$
\begin{aligned}
\left|U\left[y_{0}\right](s)\right| & =p^{-1}(0)\left|y_{2}^{\prime}(1, s)+i s h y_{2}(1, s)\right|\left|\int_{0}^{1} y_{1}(t, s) F(t, s) d t\right| \\
& \leq c_{1}|s|(1+|h|) m_{p}^{-1}\left|\int_{0}^{1} y_{1}(t, s) \rho(t)[i(g(t)+2 d(t) f(t))-s f(t)] d t\right| .
\end{aligned}
$$

Using (2.30), the inequalities (3.43) and the fact $\left\|y_{1}\right\|_{C} \leq\left\|y_{1}^{\prime}\right\|_{L_{2}}$, we have

$$
\begin{aligned}
\left|\int_{0}^{1} y_{1}(t, s) \rho(t) f(t) d t\right| \leq & 3 M_{p} s^{-2}\left\|y_{1}^{\prime}\right\| C\left\|f^{\prime}\right\|_{L_{2}}+s^{-2} M_{\rho}\|q\|_{L_{1}}\left\|y_{1}\right\|\left\|_{C}\right\| f^{\prime} \|_{L_{2}} \\
& +2|s|^{-1} M_{\rho}\|d\|_{L_{1}}\left\|y_{1}\right\|\left\|_{C}\right\| f^{\prime} \|_{L_{2}} \\
\leq & c_{1} s^{-2}\left\|f^{\prime}\right\|_{L_{2}}\left(3 M_{p}+M_{\rho}\|q\|_{L_{1}}+2 M_{\rho}\|d\|_{L_{1}}\right)
\end{aligned}
$$

which yields with $M_{2}=3 M_{p}+M_{\rho}\|q\|_{L_{1}}+4 M_{\rho}\|d\|_{L_{1}}$

$$
\left|U\left[y_{0}\right](s)\right| \leq c_{1}^{2}(1+|h|) m_{p}^{-1}\left(\sqrt{M_{\rho}}\|g\|_{\widehat{L}_{2}}+M_{2}\left\|f^{\prime}\right\|_{L_{2}}\right) .
$$

We will now provide the estimation of $\left\|y_{0}\right\|_{C}$. Analogously to (3.46), we have

$$
\left|p^{-1}(0) y_{2}(x, s) \int_{0}^{x} y_{1}(t, \mu) F(t, s) d t\right| \leq c_{1}^{2}|s|^{-1} m_{p}^{-1}\left(\sqrt{M_{\rho}}\|g\|_{\widehat{L}_{2}}+M_{2}\left\|f^{\prime}\right\|_{L_{2}}\right)
$$

Proceeding in the same way and using the fact $|s|>1$, we obtain

$$
\left|p^{-1}(0) y_{1}(x, s) \int_{x}^{1} y_{2}(t, \mu) F(t, s) d t\right| \leq c_{1}^{2}|s|^{-1} m_{p}^{-1}\left(\sqrt{M_{\rho}}\|g\|_{\widehat{L}_{2}}+M_{2}\left\|f^{\prime}\right\|_{L_{2}}\right)
$$

The estimations (3.47) and (3.48) give

$$
\left\|y_{0}\right\|_{C} \leq 2 c_{1}^{2}|s|^{-1} m_{p}^{-1}\left(\sqrt{M_{\rho}}\|g\|_{\widehat{L}_{2}}+M_{2}\left\|f^{\prime}\right\|_{L_{2}}\right) .
$$

Combining estimations (3.42), (3.43), (3.46) and (3.49) together gives

$$
\begin{aligned}
\|u(\cdot, s)\|_{C} & \leq \frac{|h||f(1)|+\left|U\left[y_{0}\right](s)\right|}{\left|U\left[y_{1}\right](s)\right|}\left\|y_{1}(x, \mu)\right\|_{C}+\left\|y_{0}(x, \mu)\right\|_{C} \\
& \leq|s|^{-1}\left(r_{1}\left\|f^{\prime}\right\|_{L_{2}}+r_{2}\|g\|_{\widehat{L}_{2}}\right),
\end{aligned}
$$

where:

$$
\begin{aligned}
& r_{1}=c_{0} c_{1}|h|+m_{p}^{-1}\left(c_{0} c_{1}^{3}(1+|h|)+2 c_{1}^{2}\right)\left(3 M_{p}+M_{\rho}\|q\|_{L_{1}}+4 M_{\rho}\|d\|_{L_{1}}\right), \\
& r_{2}=c_{0} c_{1}^{3}(1+|h|) m_{p}^{-1} \sqrt{M_{\rho}}+2 c_{1}^{2} m_{p}^{-1} \sqrt{M_{\rho}} .
\end{aligned}
$$


Finally, let us estimate $\left\|u^{\prime}(\cdot, s)\right\|_{L_{2}}$. Thanks to (3.24), we have

$$
\begin{aligned}
u^{\prime}(x, \mu)= & -\frac{i h f(1)+U\left[y_{0}\right](s)}{U\left[y_{1}\right](s)} y_{1}^{\prime}(x, \mu)+\frac{y_{2}^{\prime}(x, s)}{p(0)} \int_{0}^{x} y_{1}(t, s) F(t, s) d t \\
& +\frac{y_{1}^{\prime}(x, s)}{p(0)} \int_{x}^{1} p^{-1}(t) y_{2}(t, s) F(t, s) d t
\end{aligned}
$$

and in the same way as in (3.50) we get

$$
\left\|u^{\prime}(\cdot, s)\right\|_{L_{2}} \leq r_{1}\left\|f^{\prime}\right\|_{L_{2}}+r_{2}\|g\|_{\widehat{L}_{2}} .
$$

According to (3.40), the estimations (3.50) and (3.52) give us for $|s|>1$

$$
\left\|\left(A_{h}-s I\right)^{-1} w\right\|_{H} \leq r\|w\|_{H}
$$

with some constant $r>0$, hence the theorem is proved.

Corollary 3.7. Let $\operatorname{Re} h>0$ and $p, d$, q satisfy assumptions (3.5), (3.6), (3.7). Moreover, let $\rho$ be as in (3.4) and of bounded variation. Then there exists a positive constant $r$ defined by (3.53) such that for any mild solution of the problem (3.11)-(3.12) with initial data $\left(v_{0}, v_{1}\right) \in H$, the following estimations are true

$\|V(t)\|_{H} \leq\|V(0)\|_{H}, \quad\|V(t)\|_{H} \leq M_{\delta}\|V(0)\|_{H} e^{-\delta t} \quad$ for all $\delta<r^{-1}, \quad t \geq 0$.

\section{Acknowledgments}

The author would like to express his gratitude to the reviewer for his valuable comments and remarks.

Open Access. This article is distributed under the terms of the Creative Commons Attribution License which permits any use, distribution, and reproduction in any medium, provided the original author(s) and the source are credited.

\section{References}

[1] Cox, C., Zuazua, E.: The rate at which energy decays in a string damped at one end. Indiana Univ. Math. J. 44(2), 545-573 (1995)

[2] Cox, C., Zuazua, E.: The rate at which energy decays in a damped string. Comm Partial Differ. Equ. 19(1-2), 213-243 (1994)

[3] Daletskii, Y.L., Krein, M.G.: Stability of solutions of differential equations in Banach space. Am. Math. Soc. (1974)

[4] Engel, K.J., Nagel, R.: One-parameter semigroups for linear evolution equations. In: Graduate Texts in Mathematics, vol. 194. Springer, New York (1999)

[5] Freitas, P.: On some eigenvalue problems related to the wave equation with indefinite damping. J. Differ. Equ. 127, 320-335 (1996)

[6] Fulton, C.T., Pruess, S.A.: Eigenvalue and eigenfunction asymptotics for regular Sturm-Liouville problems. J. Math. Anal. Appl. 188(1), 297-340 (1994) 
[7] Gekhtman, M.M.: On the asymptotic behavior of the normalized eigenfunctions of the Sturm-Liouville problem on a finite interval. Math. USSR Sbornik 61(1), 185-199 (1988)

[8] Gekhtman, M.M., Aigunov, G.A: On the problem of the estimation of the normalized eigenfunctions of the Sturm-Liouville operator with a positive weight function on a finite segment. Russian Math. Surv. 52(2), 387-435 (1997)

[9] Gekhtman, M.M., Gekhtman, M.M. Jr., Zagirov, Yu.M.: Sharp estimates for normalized eigenfunctions of the Sturm-Liouville operator on a finite interval with a continuous positive weight function. Dokl. Akad. Nauk 334(3), 268-269 (1994) (Russian); translation in Russian Acad. Sci. Dokl. Math. 49(1), 68-70 (1994)

[10] Gesztesy, F., Holden, H.: The damped string problem revisited. J. Differ. Equ. 251(4-5), 1086-1127 (2011)

[11] Gomilko, A., Pivovarchik, V.: On bases of eigenfunctions of boundary problem associated with small vibrations of damped nonsmooth inhomogeneous string. Asymptot. Anal. 20(3-4), 301-315 (1999)

[12] Gomilko, A.M., Pivovarchik, V.: Parameter dependent estimates for solutions of Sturm-Liouville equations. Methods Funct. Anal. Topol. 6(4), 26-42 (2000)

[13] Heading, J.: An Introduction to Phase-Integral Methods. Wiley, New York (1962)

[14] Henry, D.: Geometric theory of semilinear parabolic equations. Lecture Notes in Mathematics, vol. 840. Berlin (1981)

[15] Krein, M.G., Nudelman, A.A.: On some spectral properties of an inhomogeneous string with dissipative boundary condition. J. Operator Theory 22, 369-395 (1989). (in Russian)

[16] Menz, G.: Exponential Stability of Wave Equations with Potential and Indefinite Damping. Konstanzer Schriften in Mathematik und Informatik Nr. 224 (2007)

[17] Muñoz Rivera, J.E., Racke, R.: Exponential stability for wave equations with non-dissipative damping. Nonlinear Anal. 68, 2531-2551 (2008)

[18] Pivovarchik, V.: Inverse problem for a smooth string with damping at the end. J. Oper. Theory 38, 243-263 (1997)

[19] Rzepnicki, E.: Generating the exponentially stable $C_{0}$-semigroup in a nonhomogeneous string equation with damping at the end. Opuscula Mathematica 33(1), 175-186 (2013)

[20] Shubov, M.A.: Asymptotic and spectral analysis of non-selfadjoint operators generated by a filament model with a critical value of a boundary parameter. Math. Meth. Appl. Sci. 26, 213-245 (2003)

[21] Shubov, M.A.: Asymptotics of resonances and geometry of resonance states in the problem of scattering of acoustic waves by a spherically symmetric inhomogeneity of the density. Differ. Integr. Equ. 8(5), 1073-1115 (1995)

[22] Shubov, M.A.: Basis properties of eigenfunctions of nonselfadjoint operator pencils generated by the equation of nonhomogeneous damped string. Integr. Equ. Oper. Theory 25, 289-328 (1996)

[23] Shubov, M.A.: Nonselfadjoint operators generated by the equation of a nonhomogeneous damped string. Trans Am. Math. Soc. 349(11), 4481-4499 (1997)

[24] Zettl, A.: Sturm-Liouville Theory. Mathematical Surveys and Monographs. AMS, Providence (2005) 
Łukasz Rzepnicki

Faculty of Mathematics and Computer Science, Nicolaus Copernicus University,

ul. Chopina 12/18, 87-100 Toruń,

Poland

e-mail: keleb@mat.umk.pl

Received: February 21, 2013.

Revised: June 3, 2013. 